\title{
PERFORMANCE EFFECTS OF STRUCTURAL AND BEHAVIOURAL DEBRIEFINGS IN TRANSPARENT SIMULATIONS
}

\author{
Carlos Capelo $^{1}$, Renato Pereira ${ }^{2}$ and João Ferreira Dias $^{3}$ \\ ${ }^{1}$ Universidade Europeia, Estrada da Correia, 53, 1500-210 Lisboa, Portugal \\ ${ }^{2}$ Iscte Business School, Instituto Universitario de Lisboa, Avenida das Forças Armadas, 1649-026 Lisboa, Portugal \\ ${ }^{3}$ Independent researcher, Rua Luis Pedroso de Barros, 26, 1400-238 Lisboa, Portugal
}

\begin{abstract}
Simulation-based learning environments are used extensively to support learning in complex business systems. Nevertheless, studies show that subjects have great difficulty in understanding and managing dynamic systems, mainly due to cognitive processing difficulties. Previous research has addressed some aspects of model transparency and instructional strategy and produced inconclusive results about their impact on learning effectiveness. This study explores the learning and performance effects of using transparent simulations (that is, showing users the internal structure of models) and applying structural and behavioural debriefings focussed respectively on the critical variables and relations and on the relation between model structure, patterns of actions, and system behaviour. We present a set of hypotheses about the influence of the model transparency and debriefing conditions on participants' performance and comprehension of the system dynamics. In a simulation experiment, participants interacted with a system dynamics model, representing the growth of a business venture. Participants provided with the more transparent strategy and exposed to structure and behavioural debriefings achieved higher performance and demonstrated better comprehension of the model dynamics. However, our findings indicate that while these conditions are beneficial for understanding the business system dynamics, making only the simulator transparent, even combined with structural debriefing, is more limited in its effect. Three out of the six hypotheses were confirmed. Structural debriefing (in conjunction with model transparency) positively influences performance, behavioural debriefing (combined with model transparency) positively influences both model dynamics comprehension and performance.
\end{abstract}

\section{KEYWORDS}

Management Education, Simulation-Based Learning, System Dynamics, Model Transparency, Simulation Debriefing

\section{INTRODUCTION}

Simulation-based learning environments (SBLEs) are promising tools for teaching in the business management domain. However, whilst the benefits of SBLEs are often discussed in the literature, evidence regarding the impact of their utilisation remains scarce. Furthermore, a need exists for more research addressing how the learning potential of such environments might be enhanced (Davidsen \& Spector, 2015). According to the literature, the effectiveness of SBLEs depends on many factors related to the learning context, e.g., simulation task, prior knowledge, interest, instructional method, and type of assessment. The present study focusses on two aspects: simulator model transparency and simulation debriefing.

Simulator transparency refers to the extent the structure of the underlying computational model is shown to students using the simulation. In black-box model or opaque simulations, students can explore a system's behaviour, but the underlying computational models remain hidden and can only be inferred by what appears on the screen. Studies have suggested that this type of "black-box" situation could lead students to form wrong mental models, interfering with proper learning. "Glass-box model" or "transparent" simulations have alternatively been proposed to obviate the above-described problems, as the relations among their variables are accessible to students. Transparent simulators have been used in system dynamics (SD) learning environments, detailing through their stocks-and-flows diagram (SFD) the causal structure of the underlying system. By using this approach, students may trace the cause and effect structure and understand the 
relationships between structure and behaviour, and they are in a position of understanding even counterintuitive system behaviours (Groesser, 2012).

Research shows that structural knowledge provided by transparent simulations has the potential to improve leaning and task performance (Größler et al., 2000). Kopainsky and Alessi (2015) found that participants provided with the more transparent strategy demonstrated better understanding of the underlying model, but their performance, however, was equivalent to those in the less transparent situation. More recently, Capelo and Silva (2020) analysed the learning effects of using transparent simulations (that is, showing participants the model SFD) and exploratory guidance (that is, guiding learners so they are able to explore the simulation by themselves) and found that while the transparent strategy combined with the exploratory guidance is a beneficial method for understanding both model structure and behaviour, making the model transparent alone is more limited in its effect. The implication of the previous studies is that students must not only identify the structure of the simulator model but also have to recognise the relationship between structure and behaviour.

According to many scholars, debriefing is a critical step in any SBLE. A debriefing session is characterized as a facilitated or guided reflection in the experiential learning cycle, structured around a set of questions that encourages students to reflect on their simulation experience. The debriefing method may be incorporated in the instructional support, by asking and discussing with students (at the end or between simulation runs) their difficulties related to the comprehension of the model structure and behaviour. Such approach can motivate learners to reflect on the simulation experience in the quest to comprehend the game behaviour and its causes. In other words, debriefing may help learners to overcome misconceptions about dynamic and complex tasks and make it possible to improve mental model construction thereby (Qudrat-Ullah, 2014). Thus, debriefing influences the potential of SBLEs in such way that students may improve their task performance and learning in dynamic tasks.

Previous research on debriefing in simulation studied the effects on learning and performance (Qudrat-Ullah, 2014) and discussed how to design debriefing sessions (Grund \& Schelkle, 2019). In the simulation experiments conducted by Qudrat-Ullah (2014), in order to facilitate the comprehension of the structure and behaviour of the underlying model, students were involved in different combinations of pre-task, in-task, and post-task discussions. Those discussions generally improved students' mental models and task performance. However, subjects provided only with pre-task facilitation performed poorly. Grund and Schelkle (2019) tested two different versions of a simulation game: one integrating debriefing into the game itself, whereas the other version used classical post hoc debriefing. Results indicate that it is favourable to integrate debriefing into the game in terms of motivation and learning outcomes.

\section{RESEARCH HYPOTHESES}

This study investigates whether model transparency combined with an instructional approach that includes structural and behavioural debriefing can improve the learning potential for teaching the dynamics of venture growth, leading to enhanced student understanding of their main concepts and performance.

Under a model-based learning perspective, students use the simulation program to form an initial mental model and develop it into a target conceptual model (the same one underlying the simulation model). Thus, the learnings outcomes refer to the students' understanding of the simulated system, in terms of structure and behaviour, and to their performance in the simulation task.

The analysis of subjects' learning is based on their actions related to specific dynamic aspects of the simulator model. As students deal with the simulator dynamics, (they interpret the situation and mentally simulate the consequences of selected actions) they define and implement courses of action which reflect their comprehension about the structure and behaviour of the simulator model.

The expected relations and hypotheses are based on the following variables: (i) Level of model transparency (LMT). This variable represents the transparency level of the simulator. LMT indicates the extent to which students have access to the variables and relations included in the simulation model when they perform the simulation task. (ii) Level of structural debriefing (LSD). This variable indicates in what extent the instructional method includes a debriefing session focussed on the variables and relations of the simulation model. (iii) Level of behavioural debriefing (LBD). This variable indicates in what extent the instructional method includes a debriefing session focussed on the behaviour of the simulation model and 
relation to its structure. (iv) Comprehension of model dynamics (CMD). This variable reflects how students comprehend the structure of the simulation model (representing the growth of a business venture) and are able to infer its dynamical behaviour. (v) Performance. The performance of this simulation task is measured in terms of the financial value created by the venture.

The following hypotheses are defined: H1 - LMT positively influences CMD, H2 - LMT positively influences Performance, H3 - LSD positively influences CMD, H4 - LSD positively influences Performance, H5 - LBD positively influences CMD, and H6 - LBD positively influences Performance.

\section{METHOD}

\subsection{Simulator, Participants, and Procedure}

The SBLE incorporates the same mathematical model based on SD that was used in previous research (Capelo et al., 2018), which represents the growth of a business venture. By using this SBLE, students would be able to appreciate the dynamical complexity involved in a business venture and the performance effects caused by system misconceptions. The simulator provides two alternative interfaces. One represents an opaque simulator and the other a transparent one. The interface includes six screens. The first screen (the panel control) allows participants to adjust simulation parameters and includes tables presenting critical measures. In the transparent interface, the panel control provides the information included in a stock and flow diagram. The second and third screen present the historical behaviour over time for key variables. The other screens, only available in the transparent interface, show the causal loop diagram (CLD) and selected SFDs of the model.

Participants run a realistic simulator of a $\mathrm{n}$ business venture by making critical decisions every six months until the end of the third year and every year onwards for a simulation period of ten years. They analyse the business status and make decisions related to the development of key resources. The participant objective was to develop those critical and interrelated resources at appropriate rates and levels in order to maximize value creation.

In order to test the hypotheses, we conducted a laboratory experiment using three treatment groups: a group (A) of students who interacted with an opaque simulator and did not participate in debriefing sessions - low LMT, low LSD, and low LBD; a group (B) of students who used a transparent simulator and did not participate in debriefing sessions - high LMT, low LSD, and low LBD; and a group (C) who used a transparent simulator and participated in debriefing sessions - high LMT, high LSD (in the second and third simulation runs), and high LBD (in the third simulation run).

This research was conducted at Iscte Business School in Lisbon, Portugal. The experiment involved three classes of entrepreneurship courses with 67 students in total. One of the authors acted as instructor in these classes. Each of the three different treatments was assigned randomly to one of the three classes: groups A (with 23 students), B (with 22 students), and C (with 22 students). As participants from group A were submitted to the basic conditions (low LMT, low LSD, and low LBD), this group was assigned the role of control group. Participants did not know the business game and had no previous experience with the simulator.

The present experiment was carried out individually in class with one participant per computer. All participants were given a full experimental guide including: description and objective of the simulation task; case text; instructions for accessing and starting the simulator; instructions for interacting with the simulator; simulation guide; and instructions for interpreting CLDs and SFDs (only for participants using a transparent simulator). The decisions made on the simulation and its results were automatically stored in a protected spreadsheet on the participant's computer.

To succeed in this simulation task, participants needed to identify and understand the cause-and-effect relationships among critical variables, particularly those included in the CLD and selected SFDs provided in the transparent interface. That model complexity has been demonstrated to negatively influence both formation of accurate mental models and tasks. Moreover, subjects who are not familiarized with systems dynamics have additional difficulties in recognizing and comprehending dynamical structures as they are not able to properly read and interpret causal-loop-diagrams and stocks-and-flows diagrams (Alessi, 2000; Davidsen \& Spector, 2015). In order to obviate these problems, we complemented model transparency with 
structural and behavioural debriefings. The students from group $\mathrm{C}$ participated in a debriefing session (after the first simulation run) focused on the model variables and relations. Then (before the third simulation run) they were involved in discussions about the relation between model structure, courses of action, and behaviour.

The experiment procedure involved two sessions and had the following steps (Figure 1).

Session 1. This session involved only the participants from experimental groups B and C. The literature (Groesser, 2012) pointed out that the extra information provided by the transparency of SD models can only benefit learners who are able to read and interpret stocks-and-flows diagrams (SFDs). Thus, the students received a lecture on CLDs and SFDs, so that they were able to read and interpret the CLD and SFDs available in the simulator interface.

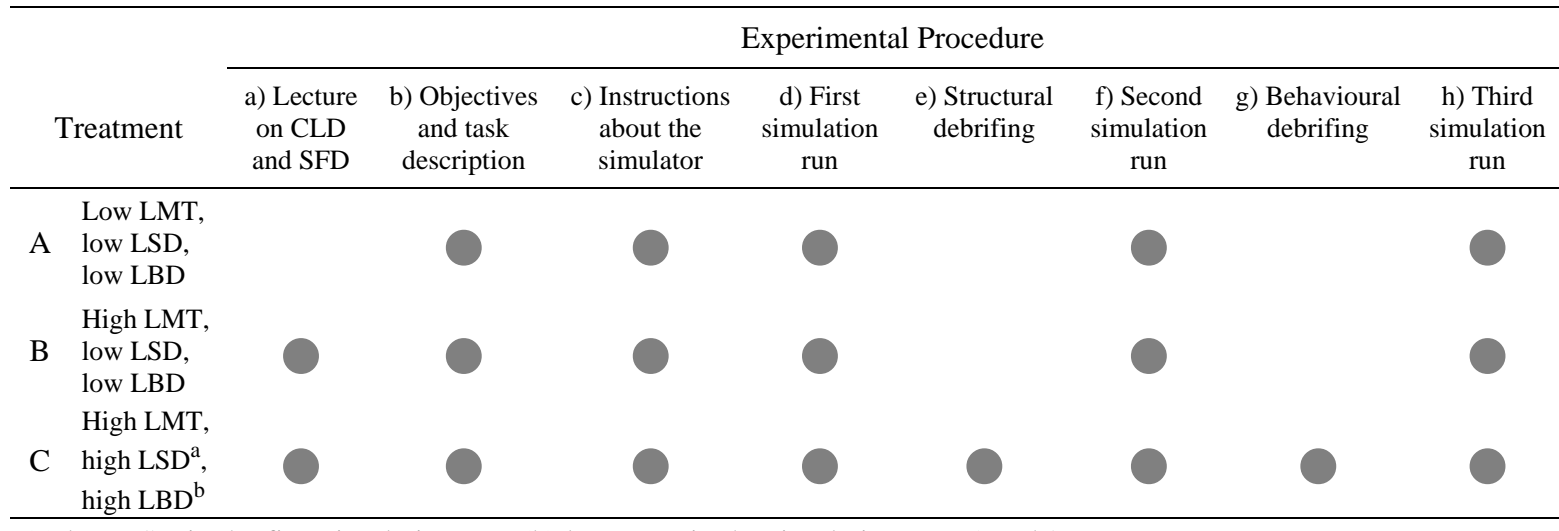

(a) low LSD in the first simulation run; (b) low LBD in the simulation runs 1 and 2

Figure 1. Experimental procedure

Session 2. In this session, students performed the simulation task using three simulation runs. They read the introduction with the overall description and the objectives of the simulation task. Participants then read the instructions for accessing, starting and running the simulator. Some simulation rounds were conducted to familiarised participants with the game interfaces and commands. In groups A and B, the three simulation runs were performed sequentially (for approximately 20 to 25 minutes each). During the simulation task, the participants from experimental groups B and C were encouraged to read and interpret the CLD and SFDs available in the game interface.

Participants from group C, after performing the first simulation run, participated in a structural debriefing (lasting approximately 35 minutes). The instructor described the CLDs and SFDs in the game interface in a form of a step-by-step guided tour and cleared all the doubts raised by students concerning the model variables and their relations. Participants then performed the second simulation run followed by a behavioural debriefing. This debriefing (lasting approximately 50 minutes) focused on the relation between the model structure, courses of action, and corresponding dynamical behaviour. The instructor first described the main challenge of this simulation task, which is how to successfully manage the growth of the venture in order to overcome the dead valley of negative financial performance and start a value creation process. The reference modes for the critical variables and corresponding desirable and "fear" scenarios were explained. Then, a debate on how certain model structures (included in the model CLD and SFDs) can support a sustainable growth was moderated by the instructor. Finally, students from group $\mathrm{C}$ performed the third simulation run.

\subsection{Research Variables}

This section summarises the use of the variables that were defined in the research model.

Level of model transparency (LMT). This variable features two degrees. In the low degree (low LMT), students perform the simulation task without accessing any structural information of the simulator model. In the high degree (high LMT), students, during the simulation task, have access to the CLD and selected SFDs of the simulator model. 
Level of structural debriefing (LSD). This variable features two degrees. In the low degree, students perform the simulation task without prior structural debriefing. In the high degree, students have participated previously in a structural debriefing.

Level of behaviour debriefing (LBD). This variable features two degrees. In the low degree, students perform the simulation task without prior behavioural debriefing. In the high degree, students have participated previously in a behavioural debriefing.

Comprehension of the model dynamics (CMD). This variable indicates how students understand the structure and behaviour of the simulation model. The measurement of this variable is based on students' patterns of action throughout the simulation task, by analysing how they consistently deal with certain dynamical structures of the simulation model. This variable is calculated as the average of four components that are rated in a continuous scale (from 0 to 1 ) against reference values.

Performance. The performance of this simulation task is measured in terms of the financial value created by the venture, indicated by the final value of the parameter MVA (market value added). This variable is rated in a continuous scale (from 0 to 1 ) against reference values of MVA.

\section{RESULTS AND DISCUSSION}

Table 1 presents the mean values, standard deviations, and sample sizes for the variables CMD and Performance corresponding to the three experimental treatments. Table 2 presents the results of a paired-samples t-test of significance for differences in means between pairs of simulation runs within treatment groups. The differences in means for the pair SR3-SR1 (which compares the mean values of the third to the first simulation run) are significant for all the three treatment groups, suggesting that, on average, all the treatment groups enhance CMD and Performance as they progress in the task, from the first to the last simulation run. A similar conclusion can be derived from the results found for the pair SR2-SR1 (which compares the second to the first simulation run). However, the differences in means for the pair SR3-SR2 are only significant for group $\mathrm{C}$ (transparent simulator with debriefings), which means that, on average, the groups A (opaque simulator) and B (transparent simulator without debriefing) do not significantly improve their CMD and Performance from the second to the third simulation run.

Table 1. Means and standard deviations for variables CMD (comprehension of model dynamics) and Performance for each simulation run and treatment group

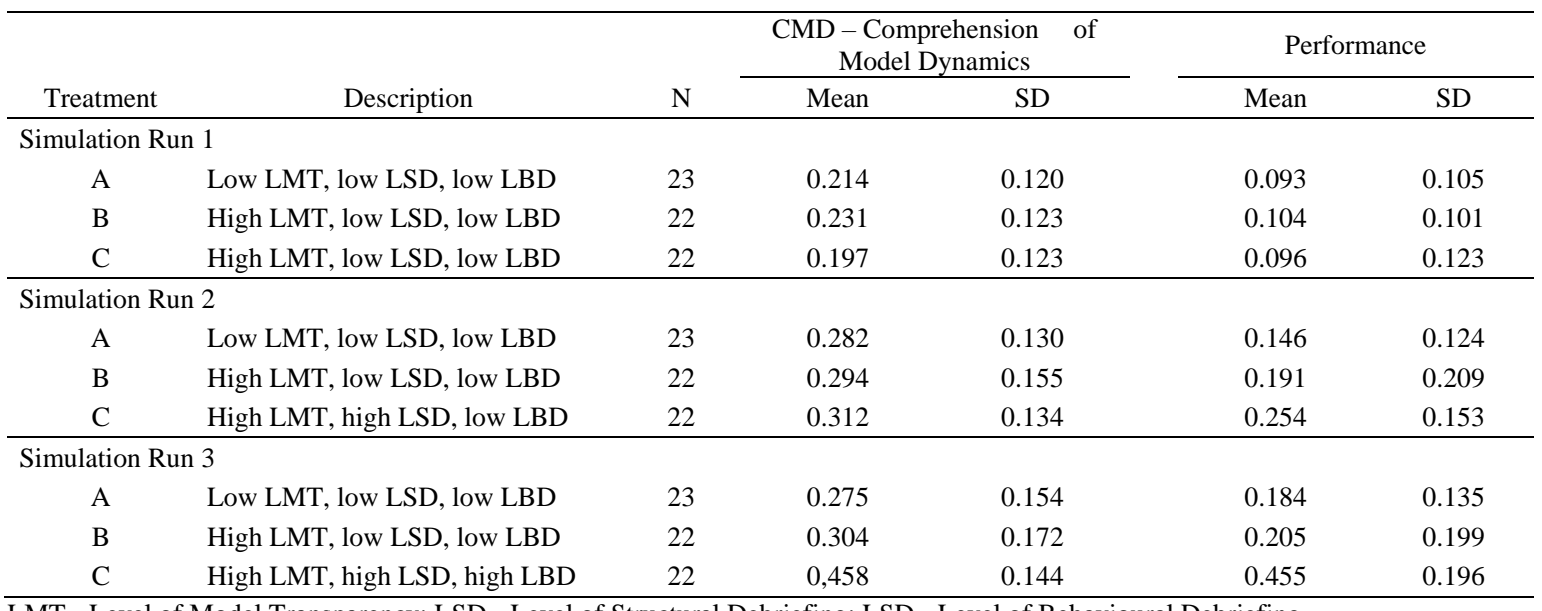

LMT - Level of Model Transparency; LSD - Level of Structural Debriefing; LSD - Level of Behavioural Debriefing.

In order to analyse and compare the degree of improvement in participants' comprehension and performance between treatment groups, we computed the variables ICMD (improvement in comprehension of model dynamics) and IP (improvement in performance), as the differences for CMC and Performance between simulation runs for each participant. Table 3 displays the results of an independent-samples t-test of significance for differences in means for the variables CMD, Performance, ICMD, and IP between pairs of treatment groups within simulation runs. 
Table 2. Paired-samples t-test of significance for differences in means for the variables CMD and Performance between pairs of simulation runs within treatment groups

\begin{tabular}{|c|c|c|c|c|c|c|}
\hline \multirow[b]{2}{*}{ Treatment } & \multicolumn{3}{|c|}{ CMD - Comprehension of Model Dynamics } & \multicolumn{3}{|c|}{ Performance } \\
\hline & Mean Difference & SD & p-value & Mean Difference & SD & p-value \\
\hline \multicolumn{7}{|c|}{ Simulation Run 2 vs Simulation Run1 } \\
\hline A & $0.069 * *$ & 0.032 & 0.044 & $0.053^{*}$ & 0.031 & 0.099 \\
\hline B & $0.063^{*}$ & 0.035 & 0.090 & $0.087 * *$ & 0.041 & 0.044 \\
\hline $\mathrm{C}$ & $0.115 * * *$ & 0.030 & 0.001 & $0.158 * * *$ & 0.043 & 0.001 \\
\hline \multicolumn{7}{|c|}{ Simulation Run 3 vs Simulation Run1 } \\
\hline A & $0.062 *$ & 0.034 & 0.081 & $0.091^{* *}$ & 0.034 & 0.013 \\
\hline B & $0.073^{*}$ & 0.035 & 0.052 & $0.101 * *$ & 0.042 & 0.025 \\
\hline $\mathrm{C}$ & $0.261 * * *$ & 0.045 & 0.000 & $0.360 * * *$ & 0.058 & 0.000 \\
\hline \multicolumn{7}{|c|}{ Simulation Run 3 vs Simulation Run 2} \\
\hline A & -0.007 & 0.023 & 0.776 & 0.038 & 0.024 & 0.124 \\
\hline B & 0.010 & 0.024 & 0.675 & 0.014 & 0.028 & 0.628 \\
\hline $\mathrm{C}$ & $0.146^{* * *}$ & 0.045 & 0.004 & $0.202 * * *$ & 0.059 & 0.003 \\
\hline
\end{tabular}

Table 3. Independent-samples t-tests of significance for differences in means for the variables CMD, Performance, ICMD, and IP between pairs of treatment groups within simulation runs

\begin{tabular}{|c|c|c|c|c|c|c|c|c|}
\hline \multirow[b]{2}{*}{ Pair } & \multicolumn{2}{|l|}{ CMD } & \multicolumn{2}{|c|}{ Performance } & \multicolumn{2}{|c|}{$\begin{array}{c}\text { Improvement in } \\
\text { CMD }\end{array}$} & \multicolumn{2}{|c|}{ Improvement in Performance } \\
\hline & Mean (SD) & p-value & Mean (SD) & $\begin{array}{c}\mathrm{p}- \\
\text { value }\end{array}$ & Mean (SD) & p-value & Mean (SD) & p-value \\
\hline B-A & $0.018(0.036)$ & 0.628 & $0.010(0.031)$ & 0.737 & $0.011(0.049)$ & 0.822 & $0.010(0.053)$ & 0,852 \\
\hline C-A & $-0.016(0.036)$ & 0.650 & $0.002(0.034)$ & 0.946 & $0.199 * *(0.056)$ & 0.001 & $0.269 * *(0.066)$ & 0,000 \\
\hline B-A & $0.012(0.043)$ & 0.785 & $0.045(0.051)$ & 0.386 & $-0.006(0.048)$ & 0.900 & $0.034(0.051)$ & 0.503 \\
\hline C-A & $0.030(0.039)$ & 0.447 & $0.107 *(0.041)$ & 0.013 & $0.047(0.044)$ & 0.294 & $0.105 *(0.052)$ & 0.050 \\
\hline C-B & $0.019(0.044)$ & 0.673 & $0.063(0.055)$ & 0.261 & $0.053(0.046)$ & 0.261 & $0.071(0.059)$ & 0.236 \\
\hline \multicolumn{5}{|c|}{ Simulation Run 3} & \multicolumn{4}{|c|}{ Simulation Run 3 vs Simulation Run 2} \\
\hline
\end{tabular}

** $\mathrm{p}<0.01, * \mathrm{p}<0.05$

The lowest mean values for the variables CMD and Performance were found in the first simulation run. As displayed in Table 1 and Table 3, the mean values for those variables are not significantly different between treatment groups. That means that participants from groups $\mathrm{B}$ and $\mathrm{C}$, with access to information concerning the structure of the simulator model, did not show better model comprehension and performance. This result may be explained by the lack of experience with the simulator. In order to start the process of building and calibrating their mental models about the simulated system, students need to complete a first simulation run. These results are somehow consistent with the findings reported by Qudrat-Ullah (2014) that subjects only submitted to pre-task facilitation perform poorly. In simulation run 2, participants in group C showed on average the best CMD (mean=0.312) and the best Performance (mean=0.254), but, according to Table 3, only the mean Performance value is significantly different from the equivalent value for group A. For the simulation run 3 , the best values for the variables CMD (mean=0.458) and Performance (mean=0.455) were also exhibited by participants from group C. The statistical testing presented in Table 3 provides evidence that the average results from group $\mathrm{C}$ are significantly different from the equivalent values for groups A and B. These results suggest that students learn and perform more effectively if the simulation approach combines model transparency with structural and behavioural debriefings. These processes combined gave participants from group $\mathrm{C}$ a significant cognitive aid that accelerated their learning about the relationships between structure and behaviour of the simulated system, thus resulting in an improved performance. 
As shown in Table 3, groups A and B show similar improvements from the first to the third simulation run, as the differences in means for the variables ICMD and IP are not significant. These results indicated that by increasing only transparent simulation, students did not learn and perform more effectively.

As presented in Table 2, participants from group $\mathrm{C}$ show, on average, the highest improvements in CMD and Performance as they progress in the task throughout the simulation runs. Additionally, the following results can be observed in Table 3. (i) From the first to the second simulation run, the differences in means for ICMD between groups are not significant, and a significant difference in means for IP is only found between group $\mathrm{C}$ and group $\mathrm{A}$. These findings indicate that, on average, participants who combined model transparency with structural debriefing improved more their performance than those using the opaque simulation. Consequently, the variable LSD seems to positively moderate the impact of LMT on Performance. (ii). From the second to the third simulation run, the differences in means for ICMD and IP between group $\mathrm{C}$ and other groups are significant, while the differences in means for those variables between groups $\mathrm{A}$ and $\mathrm{B}$ are not significant. These results suggest that, on average, participants who combine model transparency with structural and behavioural debriefings improved more their model comprehension and performance than those using only transparent simulation. Consequently, the variable LBD seems to positively moderate the impact of LMT on CMD and Performance.

Even though students from group B benefitted from a more transparent interface (showing the causal-loop-diagram and some stock-and-flow diagrams of the simulator model), they were not more successful than those from control group (A) in comprehending the model dynamics and performing the simulation task. On the other hand, as students from group $\mathrm{C}$ were engaged in a structural debriefing (after the first simulation run), they had the opportunity to clear their misinterpretations about the diagrams provided by the transparent interface, they improved their structural knowledge, and they were more able to identify the key variables and relations. Nevertheless, however, comparing to group B students, on average, group $\mathrm{C}$ participants did not significantly improve more their comprehension and performance. After the second simulation run, participants from group $\mathrm{C}$ were involved in a behavioural debriefing where they discussed and resolved their doubts about the relation between model structure, patterns of action, and corresponding expected behaviours. This helped them to improve even more their comprehension about the model dynamics and enhanced their performance in the simulation task.

Table 4 shows the results of multivariate regression analyses of CMD and Performance on the independent variables. The regressions were ran on standardised values for all the variables to directly compare the relative effects of each independent variable on the dependent variable. As presented in Table 4, regression analysis for CMD on the independent variables shows no significant effects for LMT and LSD, and significant effects for LBD and SR. Regression analysis of Performance shows no significant effects for LMT and significant effects for LSD, LBD, and SR.

Table 4. Regression results for all independent variables

\begin{tabular}{|c|c|c|c|c|}
\hline \multirow[b]{3}{*}{ Independent Variables } & \multicolumn{4}{|c|}{ Dependent Variables } \\
\hline & \multicolumn{2}{|c|}{ Comprehension of Model Dynamics } & \multicolumn{2}{|c|}{ Performance } \\
\hline & Standardized Beta & p-value & Standardized Beta & p-value \\
\hline LMT - Level of Model Transparency & 0.030 & 0.670 & 0.052 & 0.415 \\
\hline LSD - Level of Structural Debriefing & 0.122 & 0.175 & $0.207 *$ & 0.013 \\
\hline LBD - Level of Behavioural Debriefing & $0.212 *$ & 0.019 & $0.255^{* *}$ & 0.002 \\
\hline SR - Simulation Run & $0.215^{* *}$ & 0.003 & $0.228^{* *}$ & 0.001 \\
\hline Adjusted $\mathrm{R}^{2}$ & \multicolumn{2}{|l|}{0.187} & \multicolumn{2}{|l|}{0.314} \\
\hline
\end{tabular}

$* * \mathrm{p}<0.01, * \mathrm{p}<0.05$

\section{CONCLUSION}

This study is based on an educational experiment aimed at testing hypotheses about the impact of simulator transparency and debriefing sessions on students' learning and task performance. The simulation experience involves a simulator based on SD, designed to teach the dynamics of venture growth.

It was predicted that (1) making the simulator more transparent (by showing students the causal-loop and stocks-and-flows diagrams of the simulator model) and (2) providing debriefing sessions focussed on the structural (discussions about the variables and relations included in the model) and behavioural (discussions about the relation between the model structure, patterns of actions, and corresponding expected behaviours) aspects of the simulation model would benefit learning and task performance. 
The results from regression analysis confirm three of the six hypotheses. The hypotheses H1 (the level of transparency of the simulation model positively influences the level of comprehension of the model dynamics) and $\mathrm{H} 2$ (the level of transparency of the simulation model positively influences performance) are not supported, thus suggesting that only by showing students the model diagrams, they do not learn and perform any more effectively than they would otherwise. We can conclude that a simulator does not become more transparent to subjects just by showing the model diagrams. As point out by Groesser (2012), the extra information provided by the transparency of SD models can only benefit learners who are able to read and interpret SFDs. Thus, one possible explanation is that as participants were not familiarized with the system dynamics approach (even though they have received a lecture on CLDs and DFDs), they were not completely enabled and motivated to read and interpret the model diagrams.

Executing the task with a transparent simulator after being submitted to a structural debriefing session significantly improved the students' performance, supporting hypothesis H4 (The level of structural debriefing positively influences performance). This finding is consistent with some of the literature on learning from transparent models. For example, Grobler et al. (2000) also reported that a presentation about the structure of a business simulator improved participants ability to control the system and had a positive influence on their task performance. However, our research does not provide support of hypotheses H3 (the level of structural debriefing positively influences the level of comprehension of the model dynamics). As participants enhanced their knowledge on the model variables and relations, they took more advantage of the more structured information provided by the transparent interface (e.g., the stock levels and flow rates in the prospect pipeline) and improved their ability to control and manage the task, which led to better performance. Nevertheless, however, courses of action of students submitted to that condition did not show that they consistently improved their comprehension of the model dynamics. This result is consistent with the findings reported by Capelo and Silva (2020) that subjects submitted to transparent conditions are able to acknowledge certain cause-and-effect relations, but they fail to mentally infer the model behaviour. This conclusion is in line with a learning difficulty frequently mentioned in system dynamics literature (Davidsen \& Spector, 2015): that it is difficult to develop an understanding of how the behaviour of a complex system emerges from its underlying causal structure.

Regression results support hypothesis H5 (the level of behavioural debriefing positively influences the level of comprehension of the model dynamics) and hypothesis H6 (the level of behavioural debriefing positively influences performance). As we hypothesised, the results strongly show that the behavioural debriefing gave participants a powerful mean to reflect on the relation between model structure and behaviour, which accelerated their learning about the dynamics of the simulated system and enhanced performance. These results reinforce an assumption already articulated in previous research (Kopainsky \& Sawicka, 2011; Qudrat-Ullah, 2014) that is important to provide facilitation in order to improve subject's performance and their understanding of system dynamics.

\section{REFERENCES}

Capelo, C., Dias, J., \& Pereira, R. (2018). A system dynamics approach to analyse the impact of energy efficiency policy on ESCO ventures in European Union countries: a case study of Portugal. Energy Efficiency, 11, 1-33.

Capelo, C. \& Silva, A. (2020). Optimizing the learning potential of simulations through structural transparency and exploratory guidance. Simulation \& Gaming.

Davidsen, P. \& Spector, J. (2015). Critical Reflections on System Dynamics and Simulation/Gaming. Simulation \& Gaming, 46, 430-444.

Größler, A., Maier, F. H., \& Milling, P. M. (2000). Enhancing learning capabilities by providing transparency in business simulators. Simulation \& Gaming: An International Journal, 31, 257-278.

Groesser, S. N. (2012). Model-Based Learning with System Dynamics. In N. Seel (Ed.), Encyclopedia of the sciences of learning, pp 2303-2307. New York: Springer.

Grund, C. \& Schelkle, M. (2019). Developing Serious Games with Integrated Debriefing. Bus Inf Syst Eng, 62, 87-101.

Kopainsky, B. \& Alessi, S. M. (2015). Effects of Structural Transparency in System Dynamics Simulators on Performance and Understanding. Systems, 3, 152-176.

Kopainsky, B. \& Sawicka, A. (2011). Simulator-supported descriptions of complex dynamic problems: experimental results on task performance and system understanding. System Dynamics Review 27 (2), 142-172.

Qudrat-Ullah, H. (2014). Yes we can: Improving performance in dynamic tasks. Decision Support Systems 61, 23-33. 